\title{
Enhancing Cognition after Stress with Gene Therapy
}

\author{
Andrea Nicholas, ${ }^{1}$ Carolina D. Munhoz, ${ }^{1,2}$ Deveroux Ferguson, ${ }^{1}$ Laura Campbell, ${ }^{1}$ and Robert Sapolsky ${ }^{1,3}$ \\ ${ }^{1}$ Department of Biological Sciences, Stanford University, Stanford, California 94305, ${ }^{2}$ Department of Pharmacology, Institute of Biomedical Science, \\ University of São Paulo, 05311-970 São Paulo, Brazil, and ³Department of Neurology and Neurological Sciences, Stanford University School of Medicine, \\ Stanford, California 94305
}

Hippocampal function is essential for the acquisition, consolidation, and retrieval of spatial memory. High circulating levels of glucocorticoids (GCs), the adrenal steroid hormones secreted during stress, have been shown to impair both acquisition and retrieval and can either impair or enhance consolidation, depending on experimental conditions. In contrast, estrogen can enhance spatial memory performance and can block the deleterious effects of GCs on such performance. We therefore constructed a chimeric gene ("ER/GR") containing the hormone-binding domain of the GC receptor and the DNA binding domain of the estrogen receptor; as a result, ER/GR transduces deleterious GC signals into beneficial estrogenic ones. We show here that acute immobilization stress, before acquisition and retrieval phases, increases latencies for male rats in a hidden platform version of the Morris water maze. This impairment is blocked by hippocampal expression of the ER/GR transgene. ER/GR expression also blocks decreases in platform crossings caused by acute stress, either after acquisition or before retrieval. Three days of stress before acquisition produces an estrogen-like enhancement of performance in ER/GR-treated rats. Moreover, ER/GR blocks the suppressive effects of GCs on expression of brain-derived neurotrophic factor (BDNF), a growth factor central to hippocampal-dependent cognition and plasticity, instead producing an estrogenic increase in BDNF expression. Thus, ER/GR expression enhances spatial memory performance and blocks the impairing effects of GCs on such performance.

Key words: BDNF; behavior; dentate gyrus; estrogen (estradiol); glucocorticoid; hippocampal function; learning and memory; memory formation; spatial cognition; spatial memory; stress

\section{Introduction}

Hippocampal-dependent spatial learning and memory can be separated into distinct phases, namely acquisition, consolidation, and retrieval (Roozendaal, 2003), and it is now recognized that hormones can modulate such processes. The effects of glucocorticoids (GCs), the adrenal steroid hormones released during stress, on spatial memory depend on GC levels and the time of GC administration relative to each phase (Roozendaal, 2003). Chronic stress $(21 \mathrm{~d}$ ) before acquisition impairs performance in the hidden platform version of the Morris water maze (MWM) (Bodnoff et al., 1995; Sousa et al., 2000; Sandi et al., 2003; Kitraki et al., 2004; Stewart et al., 2005). In contrast, 7-10 d of stress before acquisition may impair (Radecki et al., 2005) or enhance (Gouirand and Matuszewich, 2005) whereas stressors immediately before training worsen performance $24 \mathrm{~h}$ later in the MWM (Kim et al., 2001).

After acquisition, administration of GCs facilitate memory consolidation in MWM under low stress $\left(25^{\circ} \mathrm{C}\right.$ water $)$ but not high stress $\left(19^{\circ} \mathrm{C}\right.$ water or predator exposure) conditions, suggesting that moderate stress levels of GCs are beneficial (Sandi et

Received April 19, 2006; revised Sept. 26, 2006; accepted 0ct. 5, 2006

This work was supported by the Adler Foundation and National Institutes of Health Grant R01 AG020633. We thank Drs. B. McEwen, D. Diamond, and B. Roozendaal for advice on this study.

Correspondence should be addressed to Andrea Nicholas, Department of Biological Sciences, Stanford University, Gilbert Laboratory, MC 5020, Stanford, CA 94305-5020. E-mail: anichola@stanford.edu. D01:10.1523/JNEUROSCI.3122-06.2006

Copyright $\odot 2006$ Society for Neuroscience $\quad 0270-6474 / 06 / 2611637-07 \$ 15.00 / 0$ al., 1997; Diamond et al., 1999). In contrast, adrenalectomy impairs MWM acquisition (Oitzl and de Kloet, 1992; Conrad and Roy, 1993). Finally, stress or elevated GC exposure before memory testing interferes with memory retrieval, impairing performance (de Quervain et al., 1998; Roozendaal et al., 2003). Thus, collectively, whereas moderate increases in GCs can facilitate performance on spatial memory tasks, very low and high stress levels of GCs impair (Roozendaal, 2003).

In contrast with GCs, estrogen improves spatial performance (Luine and Rodriguez, 1994; Packard et al., 1996; McEwen et al., 1997; Packard, 1998; Sandstrom and Williams, 2001; Bowman et al., 2002; Frick et al., 2002) and spares stress-induced impairments (McEwen et al., 1997; Luine, 2002; Bisagno et al., 2003), effects shown in both female and male rats (Packard et al., 1996; Packard, 1998). Estrogen may exert these beneficial effects via brain-derived neurotrophic factor (BDNF) expression, because it is upregulated in the hippocampus by estrogen, is required for short- and long-term memory formation (Alonso et al., 2002), and blocks the adverse effects of stress on spatial memory (Radecki et al., 2004).

Estrogen and GCs are steroid hormones with intracellular receptors that, when activated, regulate gene expression by binding specific DNA-responsive elements (McEwen, 2001b). In the present report, we use gene therapy with a chimeric gene ("ER/GR") containing the hormone-binding domain of the GC receptor and the DNA binding domain of the estrogen receptor; this construct converts the actions of GCs at the receptor level into estrogenic 
effects at the transcriptional level, thereby converting deleterious GC effects into beneficial estrogenic ones (Kaufer et al., 2004).

A previous study examined the beneficial effects of ER/GR in the realm of an excitotoxic insult (Kaufer et al., 2004). We now investigate whether ER/GR would also be beneficial in the cognitive realm.

\section{Materials and Methods}

Subject. Male Sprague Dawley rats (Charles Rivers Laboratories, Wilmington, MA), weighing between 200 and $400 \mathrm{~g}$, were housed under standard laboratory conditions on a $12 \mathrm{~h}$ light/dark cycle with lights on at 7:00 A.M.

Vector construction and intracerebral infusion. The construction of a bipromoter herpes simplex virus-1 amplicon vector expressing the ER/GR chimeric receptor gene [along with green fluorescent protein (GFP) as a reporter] has been described previously (termed ER/GR) (Kaufer et al., 2004); the control vector expressed GFP alone (termed GFP). For stereotaxic infusion of vector, rats were anesthetized with 1 $\mathrm{ml} / \mathrm{kg}$ of a mixture consisting of $100 \mathrm{mg} / \mathrm{kg}$ ketamine, $10 \mathrm{mg} / \mathrm{kg}$ acepromazine, and $100 \mathrm{mg} / \mathrm{kg}$ xylazine. The ER/GR or GFP vectors were infused $\left(1 \mu\right.$ l over a 5 min period with a titer of $\sim 1 \times 10^{7}$ ) dorsal to the apex of the dentate gyrus (DG) (coordinates: anteroposterior, $-4 \mathrm{~mm}$ from bregma; mediolateral, 3.00 from midline; dorsoventral, $-3.2 \mathrm{~mm}$ from dura). All injections for behavioral studies were bilateral. Rats were allowed 1 full day to recover before behavioral testing.

Histology. Transgene expression peaks $24-48 \mathrm{~h}$ after injection (Kaufer et al., 2004). Therefore, rats from behavioral studies were not used to determine ER/GR expression. A separate group of rats expressing ER/GR was perfused with heparinized saline, followed by a $4 \%$ solution of paraformaldehyde $36-48 \mathrm{~h}$ after injection. Because expression level of the GFP-tagged chimeric receptor itself is not stress inducible, nonstressed rats were used to demonstrate GFP expression for both stressed and nonstressed conditions. Brains were removed, placed in 30\% sucrose overnight, sectioned at $30 \mu \mathrm{m}$, and visualized at 490 excitation to photograph GFP-positive neurons.

Morris water maze. This consisted of a black circular pool $(1.7 \mathrm{~m}$ in diameter) filled with $25-27^{\circ} \mathrm{C}$ water. A light source and patterns on the walls surrounding the pool served as extramaze cues. Rats were trained for six blocks consisting of three $(60 \mathrm{~s})$ trials separated by $20 \mathrm{~min}$ interblock intervals. A different start location was used for each trial. Immediately after the sixth block, the hidden platform was removed and rats were scored during a $60 \mathrm{~s}$ probe trial for latency to and crossings over the previous platform location. Each swim was digitally recorded and tracked using MetaMorph software (Molecular Devices, Palo Alto, CA). For acute stress studies, the platform was returned and a final trial was given to reinforce platform presence. Another probe trial was run $24 \mathrm{~h}$ after training.

Acute stress manipulations for MWM studies. On day 1 , rats were infused with control or experimental vector and were allowed $48 \mathrm{~h}$ recovery before undergoing MWM training on day 3. Rats received immobilization stress immediately before training, directly after the immediate probe trial, or immediately before the $24 \mathrm{~h}$ probe trial. Immobilization stress consisted of 90 min restraint in plastic decapicone bags secured at the base of the tail to prevent forelimb and hindlimb movement. This stressor produces circulating levels of corticosterone (the predominant GC of rats) in the medium stress range (i.e., $\sim 30 \mu \mathrm{g} / \mathrm{dl}$ ) (Sapolsky et al., 1995).

Chronic stress manipulations for MWM studies. On day 1, rats were infused with ER/GR or GFP. On days 2-4, rats received subcutaneous injection of estradiol ( $15 \mu \mathrm{g} / \mathrm{kg}$ ) or vehicle and were immediately subjected to either gentle handling or rotating stressors (daily: $1 \mathrm{~h}$ restraint for two times, $1 \mathrm{~h}$ shaking for two times, $3 \mathrm{~h}$ cold room, and brief sedation with halothane, sequence rotated daily, with $30 \mathrm{~min}$ rest between each stressor). Such stressors produce circulating corticosterone levels in the moderate to medium stress range (i.e., 15-30 $\mu \mathrm{g} / \mathrm{dl}$ ) (Sapolsky et al., 1995). Immediately after the stressors on day 4 , rats were trained on the MWM. Rats were carefully monitored during all stress procedures, which were performed per National Institutes of Health and Stanford University Department of Veterinary Services and Care guidelines.

$B D N F$ expression. Rats were stereotaxically infused with ER/GR into the dentate gyrus of the right hemisphere and GFP into the left. This was followed by $3 \mathrm{~d}$ of immobilization stress $(3 \mathrm{~h} / \mathrm{d})$ or gentle handling, after which the hippocampus was removed. The effect of stress-dependent activation of ER/GR-mediated gene expression in the right hemisphere was measured as a percentage change in BDNF mRNA levels relative to levels in the GFP-expressing left hemisphere. Semiquantitative reverse transcription (RT)-PCR amplification was performed (ThermoScript

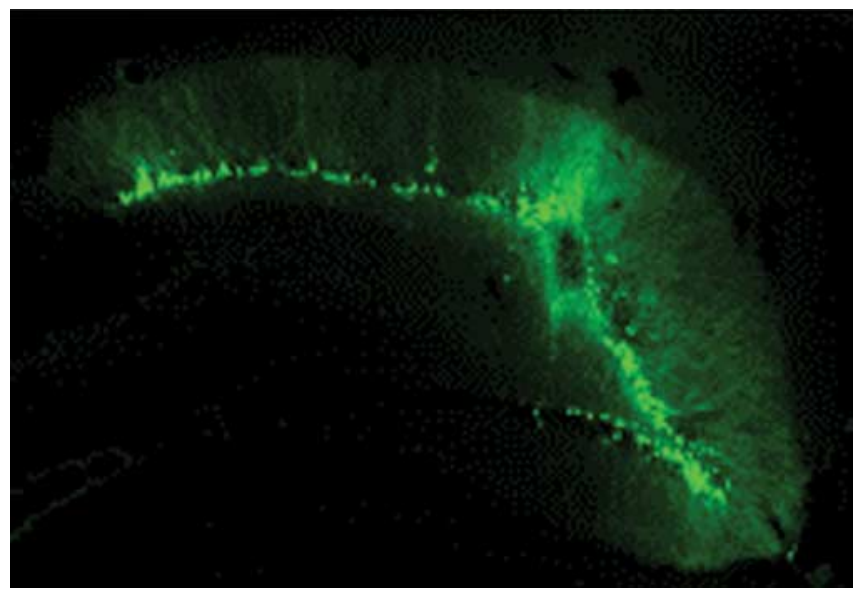

Figure 1. Typical expression of the GFP-tagged ER/GR transgene in hippocampus $48 \mathrm{~h}$ after infusion. Robust expression was observed for GFP and ER/GR vectors throughout the dentate gyrus of the dorsal hippocampus, with the vast majority in the dorsal blade.

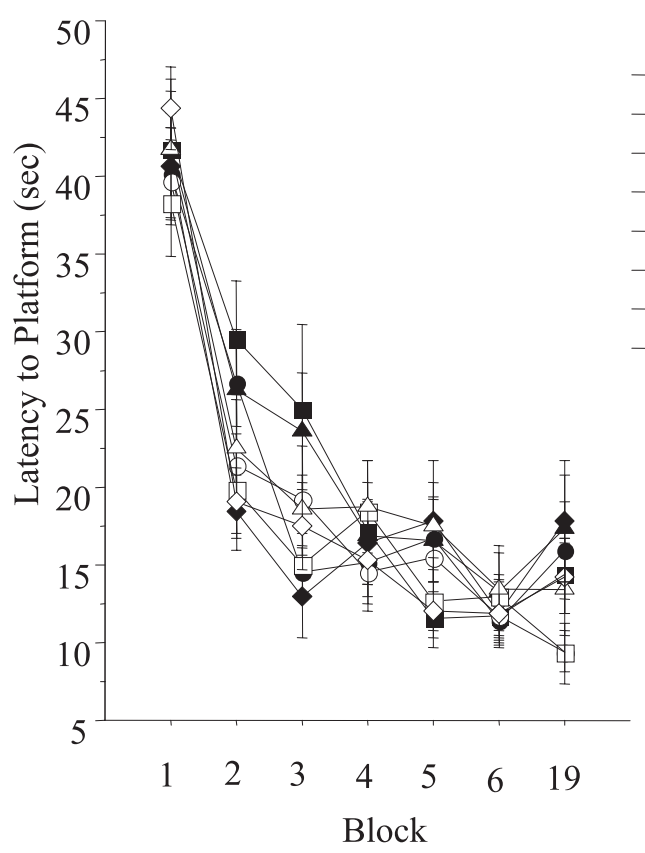

Figure 2. Learning curves for GFP- and ER/GR-expressing control rats and rats treated with stress before training, after training, and before retrieval are represented as latency to platform ( $n=9-11$ per group). There was an overall significant effect of block ( $p<0.001$ ) for all groups, with no difference between treatment groups. Mean \pm SEM in all figures. 


\section{A. $\square$ GFP}

A
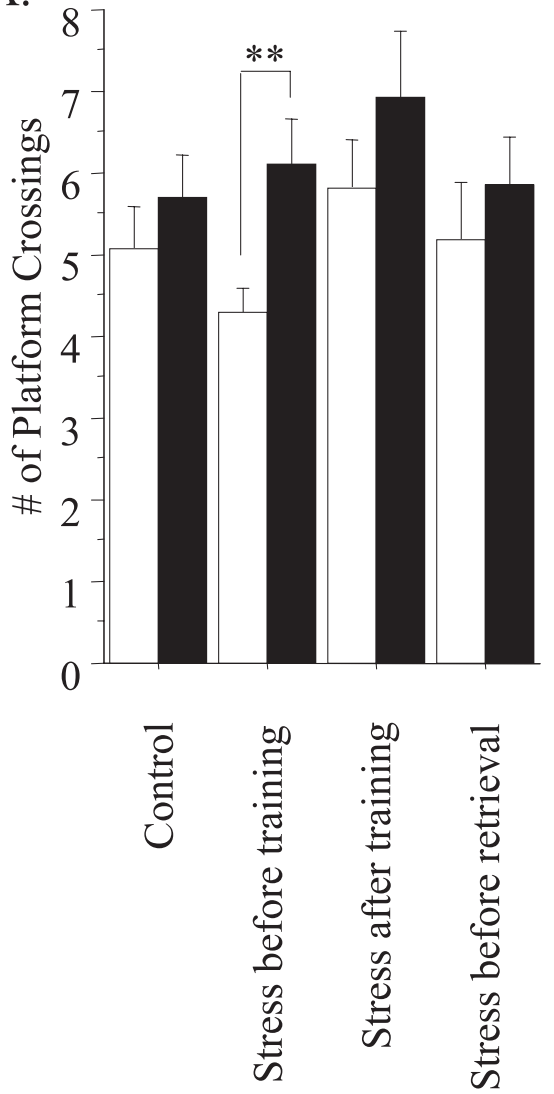

B.

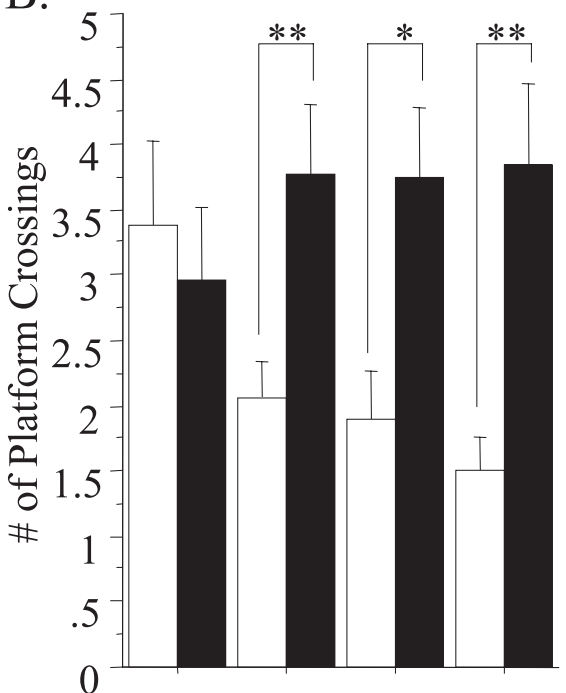

Figure 3. Platform crossing number during the immediate $(\boldsymbol{A})$ and $24 \mathrm{~h}(\boldsymbol{B})$ probe trials of the MWM. $\boldsymbol{A}$, Crossings for GFP and ER/GR rats stressed before training, after training, or before retrieval ( $n=9-11$ per group). An overall enhancing effect of ER/GR on performance was observed $(p<0.01)$. Post hoc analysis revealed a trend toward significance in "Stress before retrieval" groups ( $p=0.09)$. $\boldsymbol{B}$, An overall impairing effect of stress was observed $(p<0.0001)$ along with a significant interaction of vector and stress $(p<0.05)$. GFP rats stressed before retrieval made significantly fewer crossings than control rats $(p<0.05)$, whereas a similar trend was noted for GFP rats stressed after training $(p=0.05)$. ER/GR expression rescued the impairments in both treatment groups. Consequently, ER/GR rats made significantly more crossings when stressed after training $(p<0.01)$ and stressed before retrieval $(p<0.001)$ relative to GFP rats. A trend toward increased crossings for ER/GR rats was also observed for rats stressed after training $(p=0.09)$. Two-way ANOVA with post hoc analysis; ${ }^{*} p<0.01,{ }^{* *} p<0.001$.

RT kit; Invitrogen, Carlsbad, CA) as per the instructions of the manufacturer. BDNF primers were $5^{\prime}$-ATG CTC AGC AGT CAA GTG CC-3' (sense) and 5'-AGC CTT CCT TCG TGT AAC CC-3' (antisense). Actin was amplified as an internal PCR control using the following primers: 5'-TGA AAC AAC ATA CAA TTC CAT CAT GAA GTG TGA C-3' (sense) and 5' -AGG AGC GAT AAT CTT GAT CTT CAT GGT GCT-3' (antisense). Photographs were scanned (HP 1200 series; HewlettPackard, Palo Alto, CA), and the optic density of the bands was quantified (NIH ImageJ software).

Statistics. Rats were scored for platform crossings, latency to platform, and time spent in goal quadrant. Data for MWM experiments were analyzed using two-way repeated-measures ANOVA and two-way ANOVA with post hoc Fisher's PLSD analysis and Bonferroni's/Dunn's adjustment when necessary. The percentage increase in BDNF mRNA levels was analyzed using a within-subject one-sample analysis (95\% confidence interval). Data were analyzed with StatView (SAS Institute, Cary, NC).

\section{Results}

\section{Expression of the ER/GR transgene}

Robust expression was observed for GFP and ER/GR vectors throughout the dentate gyrus even $4 \mathrm{~d}$ after injection, with the vast majority in the dorsal blade (Fig. 1). Essentially all expression was confined to the dentate gyrus $\sim 300 \mu \mathrm{m}$ rostral and caudal of the injection site; expression in CA3 and CA1 was observed in one rat.

\section{Influence of ER/GR expression and} acute stress on platform crossings Neither stress nor the infused vector altered learning of the MWM (Fig. 2). We then examined whether ER/GR expression altered the effects of acute immobilization stress, administered at different phases of the learning and memory process, on spatial performance in the MWM, in which better performance is shown as an increase in crossings over the region of the removed hidden platform.

Stress (before training) did not alter performance in the immediate probe trial in GFP rats (Fig. 3A). An overall significant influence of vector expression on numbers of platform crossings was observed (Fig. $3 A$ ), suggesting that rats that received ER/GR vector outperformed those that received GFP. Post hoc analysis indicated that ER/GR enhanced performance in pretraining stressed rats, suggesting an influence of ER/GR expression on immediate recall.

In the $24 \mathrm{~h}$ probe trial (Fig. $3 B$ ), all stress protocols (i.e., stress before or after training, or before retrieval) impaired performance in GFP rats compared with their nonstressed GFP controls. ER/GR expression completely rescued this stressmediated impairment for all treatment groups.

\section{Influence of ER/GR expression and acute stress on latency measures} We then tested whether ER/GR would spare and/or enhance latency performance in the face of stress. Latency to platform was scored as the amount of time taken to make the first platform crossing in either the immediate or $24 \mathrm{~h}$ probe trial; thus, shorter latencies indicate better performance.

Neither stress nor vector treatment altered performance in the immediate probe trial (Fig. $4 A$ ). In the $24 \mathrm{~h}$ probe trial, GFP groups that received stress before retrieval had significantly longer latencies relative to GFP control groups (Fig. 4B). ER/GR expression completely blocked this effect. For both immediate and 24 h experiments, there was no significant influence of stress $(p>0.1)$ or vector treatment $(p>0.1)$ on swim speed (data not shown), suggesting that observed differences in performance were not the result of differences in activity level.

\section{Influence of ER/GR expression and repeated stress on MWM performance}

Because ER/GR-mediated protection was observed for rats that received $90 \mathrm{~min}$ of stress immediately before training, we next investigated whether ER/GR expression altered the effects of $3 \mathrm{~d}$ of stress before training. An additional treatment group was 
added to compare the effects of ER/GR activation with those of exogenous estrogen.

MWM acquisition data did not differ significantly between treatment groups (Fig. $5 A$ ). In GFP rats, $3 \mathrm{~d}$ of rotating stressors did not alter performance in the immediate probe trial, as assessed by numbers of platform crossings (Fig. 5B). Estradiol significantly enhanced performance under these conditions, as did stress in ER/GR-treated rats. No effect of stress or vector treatment was observed for latency or swim speed or platform crossings in the $24 \mathrm{~h}$ probe trial (data not shown).

\section{Influence of ER/GR expression on the effect of stress on BDNF expression}

We next examined the effects of stress and ER/GR at the genomic level, seeking a gene that is regulated in opposite directions by GCs and estrogen. We chose BDNF, which has been shown to protect against stressand GC-dependent spatial memory impairment (Almli et al., 2000; Schaaf et al., 2000; Alonso et al., 2002; Radecki et al., 2004). To investigate ER/GR functioning in vivo at the transcriptional level, we measured intrahippocampal BDNF mRNA expression levels after stress and mild handling. Rats were injected with ER/GR and GFP into the dentate gyrus of right and left hemispheres of the hippocampus, respectively. In stressed animals, expression of BDNF mRNA was elevated in hippocampi expressing ER/GR relative to GFP, demonstrating an estrogenlike response (Fig. 6). In the absence of stress, no difference in BDNF mRNA expression was found between hemispheres expressing ER/GR or GFP.

\section{Discussion}

There is now ample evidence that hormones can alter facets of cognition. A number of studies have focused on blocking the often-deleterious effects of GCs. For example, this has been shown pharmacologically using GC-synthesis inhibitors or GR antagonists (Oitzl et al., 1997; Sousa and Almeida, 2002). In the present study, we use a gene therapy approach to not only block the deleterious effects of stress but to convert them into protective estrogenic actions. The construction of the chimeric ER/GR was contingent on the modular nature of steroid hormone receptors, something previously exploited in the construction of the opposite receptor chimera (i.e., one with a hormone binding domain from the ER and a DNA binding domain from GR) (Green and Chambon, 1987), as well as in our previous work with ER/GR, showing its capacity to protect hippocampal neurons from acute necrotic insults (Kaufer et al., 2004).

The actions of ER/GR in the present study reflect the array of ways that GCs and stress influence spatial and recognition task performance. Whereas severe and prolonged exposure to stress and/or stress levels of GCs impair many domains of hippocampal-dependent memory retrieval, milder exposure can actually facilitate it. This "inverse-U" pattern reflects the heterogeneity of receptors for GCs in the hippocampus. The salutary
B.

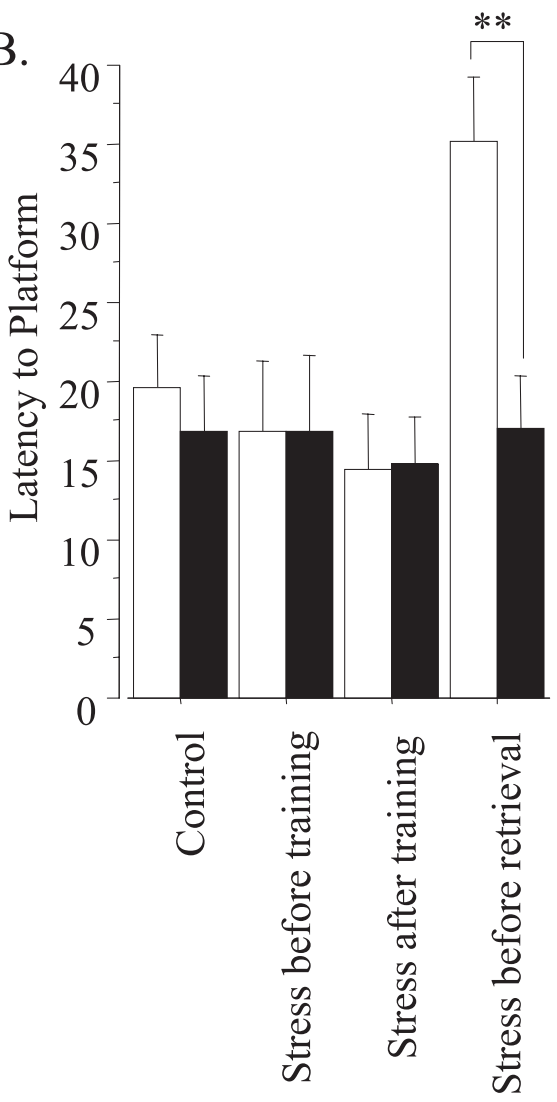

Figure 4. Latency to platform during the immediate $(\boldsymbol{A})$ and $24 \mathrm{~h}(\boldsymbol{B})$ probe trials of the MWM. $\boldsymbol{A}$, Latency to platform for GFP were observed. $\boldsymbol{B}, \mathrm{GFP}$ rats stressed before retrieval in the $24 \mathrm{~h}$ probe trial displayed significantly longer latency to platform relative to all other GFP treatment groups ( $p<0.01$ relative to all groups). This stress-induced impairment was completely rescued by ER/GR expression $(p<0.001)$. Two-way ANOVA with post hoc analysis; ${ }^{* *} p<0.01$.

effects of mild GC elevations are mediated by the high-affinity mineralocorticoid receptor (MR), whereas the disruptive effects are mediated by the low-affinity GR that is only heavily occupied in the face of high stress levels of GCs. The balance between the opposing MR and GR effects varies with the sex of the animal, pattern of GC exposure, memory task, and context of exposure relative to the different phases of learning and memory, including acquisition, consolidation, and retrieval (Sapolsky et al., 2000; McEwen, 2001a; Roozendaal, 2003). It is an oversimplification to assume that only negative stress effects are mediated by GRs. Indeed, studies have shown that moderate increases in GCs and subsequent GR activation can also facilitate the learning and memory process (Sousa and Almeida, 2002; Roozendaal, 2003).

The present findings show the impact of immobilization stress on two distinct gauges of spatial memory performance. The first, platform crossings, or the repeated seeking of the platform in its absence, revealed an impairment of acute stress administered before training, after training, and before retrieval in the $24 \mathrm{~h}$ probe trial (Fig. 3B). This robust impairment in the $24 \mathrm{~h}$ probe trial likely results from the effects of stress on the consolidation and retrieval phases along with the increased difficulty of the $24 \mathrm{~h}$ memory task relative to the immediate recall task (Fig. 3B). ER/GR expression blocks this negative effect on spatial memory, suggesting that GC-mediated activation of the chimeric receptor effectively protects against stress.

ER/GR expression also resulted in an overall enhancement of performance in the immediate probe trial (Figs. 3A, 5B). ER/GRexpressing rats that received either acute immobilization stress 
A.

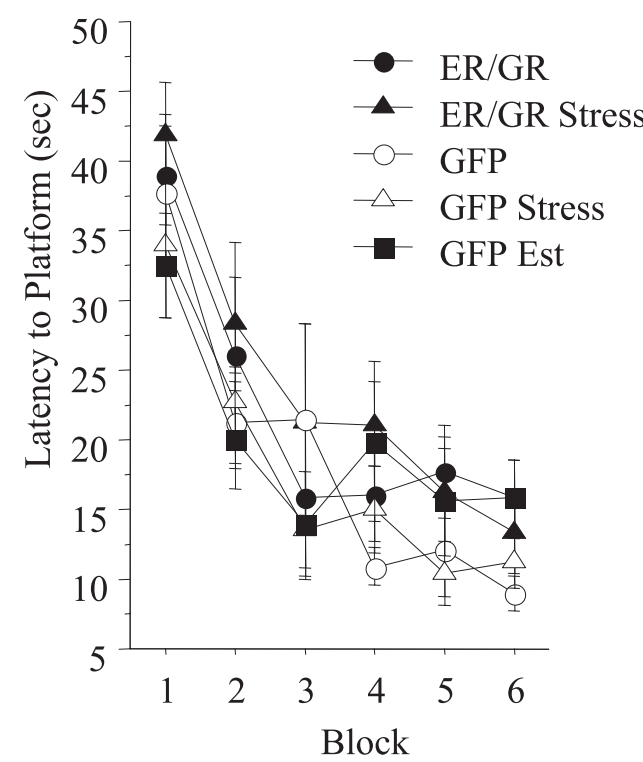

B.

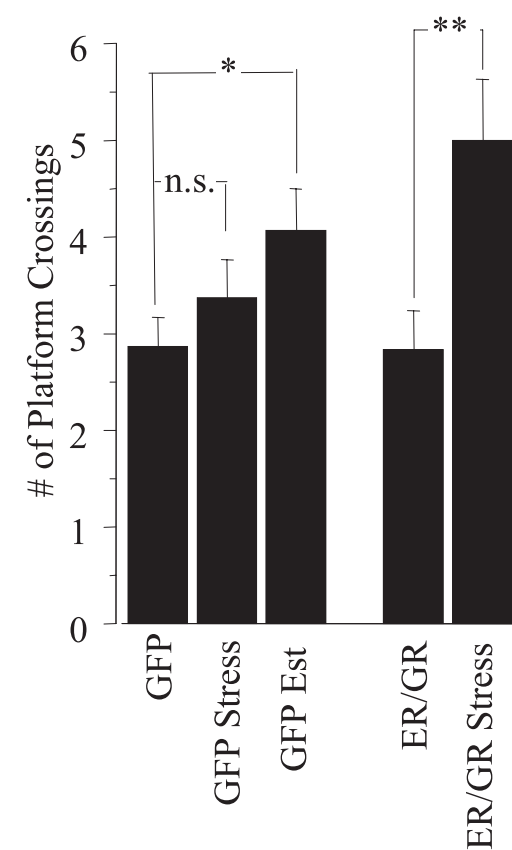

impairment, suggesting that the GCdependent protective effects of the gene therapy can occur immediately after stress (Fig. 4).

There is a wide range of mechanisms that may underlie the protective effects of ER/GR expression. After stress, an estrogenic-like transcriptional upregulation of BDNF mRNA was observed in hippocampi expressing ER/GR, showing that the chimeric receptor effectively converts a GC signal into a genomically mediated estrogenic event in vivo (Fig. 6). BDNF itself is necessary for memory formation and protects against stress-dependent impairment of spatial memory (Alonso et al., 2002; Radecki et al., 2004). Additional studies are underway to investigate whether the observed behavioral results may involve ER/GR-dependent stimulation of dendritic remodeling or synapse formation, a known effect of estrogen (Woolley, 1998).

Ultimately, ER/GR is activated by GCs via stress and functions effectively at both the behavioral and genomic levels. Although there is a strong literature supporting the theory that stress-dependent increases in GCs can modify hippocampal function and cause spatial memory impairments (Sapolsky et al., 1985; Roozendaal et al., 1997; McEwen, 1999; McGaugh and Roozendaal, 2002), we cannot state that the stress-mediated behavioral impairments observed here are definitively GC dependent. Supporting the idea of a GC role, our current work demonstrates similar protective effects using a vector that exclusively blocks GRmediated effects, without causing estrogenic ones (data not shown). However, even if the underlying cause of the impairments does not involve GCs, the GCmediated activation of the estrogenic effects of ER/GR, like BDNF upregulation, are still protective. If, conversely, the behavioral impairments are GC dependent, ER/GR-mediated protection may occur via its estrogenic effects and/or through direct competition with endogenous GR re-

(Fig. $3 A$ ) or $3 \mathrm{~d}$ of rotating stressors (Fig. $5 B$ ) before training both showed an increase in platform crossings relative to their GFP controls, suggesting that stress-dependent activation of the ER/GR chimera enhances recall of the platform location. Because no stress-mediated impairment was observed in the immediate probe trial, acquisition and recall may be less sensitive to shortterm GC effects than consolidation and retrieval.

Like platform crossings, latency (Fig. 4), the time needed for a rat to recall the exact location of the hidden platform and execute escape, is not impaired by stress in the immediate probe. Impairment is observed only when acute stress occurs before the $24 \mathrm{~h}$ probe trial, demonstrating an adverse effect on retrieval of spatial memory (Fig. 4). Intrahippocampal expression of ER/GR blocks this ceptors for circulating GCs, thereby lessening GR-mediated events in DG. In that case, the fast block ER/GR provides against the stress-induced increase in latency before retrieval may depend more on its ability to compete with endogenous GRs rather than its estrogenic genomic events. In support of this, previous work has shown latency to be a GR-dependent measure (Oitzl et al., 1997; de Kloet, 2000).

These studies are of heuristic value for understanding endocrine modulation of cognition. In addition, these studies are commensurate with those suggesting a therapeutic potential for gene therapy in the CNS. Although the majority of such studies have been oriented toward preventing neuron death after an excitotoxic insult, a few have altered behavior (for example, addic- 
tive behavior, affiliative behavior or, in the present case, the cognitive response to stress hormone exposure) (Carlezon et al., 1997; Landgraf et al., 2003). Stereotaxic microinfusion of herpes amplicon vectors at the titers used in the present study typically heavily infect cells (with a marked preference for neurons over glia) up to a few millimeters from the infusion site (Fig. 1). Although this represents only a small volume of the hippocampus, previous studies with this same vector system have demonstrated that this is sufficient to alter hippocampal-dependent behaviors (McLaughlin et al., 2000). Thus, the capacity for this vector to influence a behavior dependent on the entire hippocampus as well as other neural structures becomes that much more noteworthy.

The vectors used in this study preferentially infect granule cells of DG, and previous studies have shown the infection itself to have no impact on DG function (Dumas et al., 1999, 2000). In addition, the DG is highly responsive to stress (Bear and Abraham, 1996; Camodeca et al., 1998; Huang et al., 1999; Fujikawa et al., 2000; Ahmed et al., 2006), and modulation of DG function can readily influence spatial performance in the MWM (Conrad and Roy, 1993, 1995; Vaher et al., 1994; Xavier et al., 1999). We have shown here that gene delivery into DG can protect against stress-mediated impairment of spatial performance. This suggests that, should techniques for the safe delivery and spread of such vectors improve, gene therapy approaches might ultimately be useful for diminishing the adverse neurobiological consequences of stress.

\section{References}

Ahmed T, Frey JU, Korz V (2006) Long-term effects of brief acute stress on cellular signaling and hippocampal LTP. J Neurosci 26:3951-3958.

Almli CR, Levy TJ, Han BH, Shah AR, Gidday JM, Holtzman DM (2000) BDNF protects against spatial memory deficits following neonatal hypoxia-ischemia. Exp Neurol 166:99-114.

Alonso M, Vianna MR, Depino AM, Mello e Souza T, Pereira P, Szapiro G, Viola H, Pitossi F, Izquierdo I, Medina JH (2002) BDNF-triggered events in the rat hippocampus are required for both short- and long-term memory formation. Hippocampus 12:551-560.

Bear MF, Abraham WC (1996) Long-term depression in hippocampus. Annu Rev Neurosci 19:437-462.

Bisagno V, Bowman R, Luine V (2003) Functional aspects of estrogen neuroprotection. Endocrine 21:33-41.

Bodnoff SR, Humphreys AG, Lehman JC, Diamond DM, Rose GM, Meaney MJ (1995) Enduring effects of chronic corticosterone treatment on spatial learning, synaptic plasticity, and hippocampal neuropathology in young and mid-aged rats. J Neurosci 15:61-69.

Bowman RE, Ferguson D, Luine VN (2002) Effects of chronic restraint stress and estradiol on open field activity, spatial memory, and monoaminergic neurotransmitters in ovariectomized rats. Neuroscience 113:401-410.

Camodeca N, Rowan MJ, Anwyl R (1998) Induction of LTD by increasing extracellular $\mathrm{Ca}^{2+}$ from a low level in the dentate gyrus in vitro. Neurosci Lett 255:53-56.

Carlezon Jr WA, Boundy VA, Haile CN, Lane SB, Kalb RG, Neve RL, Nestler EJ (1997) Sensitization to morphine induced by viral-mediated gene transfer. Science 277:812-814.

Conrad CD, Roy EJ (1993) Selective loss of hippocampal granule cells following adrenalectomy: implications for spatial memory. J Neurosci 13:2582-2590.

Conrad CD, Roy EJ (1995) Dentate gyrus destruction and spatial learning impairment after corticosteroid removal in young and middle-aged rats. Hippocampus 5:1-15.

de Kloet ER (2000) Stress in the brain. Eur J Pharmacol 405:187-198.

de Quervain DJ, Roozendaal B, McGaugh JL (1998) Stress and glucocorticoids impair retrieval of long-term spatial memory. Nature 394:787-790.

Diamond DM, Park CR, Heman KL, Rose GM (1999) Exposing rats to a predator impairs spatial working memory in the radial arm water maze. Hippocampus 9:542-552.

Dumas T, McLaughlin J, Ho D, Meier T, Sapolsky R (1999) Delivery of herpes simplex virus amplicon-based vectors to the dentate gyrus does not alter hippocampal synaptic transmission in vivo. Gene Ther 6:1679-1684.

Dumas TC, McLaughlin JR, Ho DY, Lawrence MS, Sapolsky RM (2000) Gene therapies that enhance hippocampal neuron survival after an excitotoxic insult are not equivalent in their ability to maintain synaptic transmission. Exp Neurol 166:180-189.

Frick KM, Fernandez SM, Bulinski SC (2002) Estrogen replacement improves spatial reference memory and increases hippocampal synaptophysin in aged female mice. Neuroscience 115:547-558.

Fujikawa T, Soya H, Fukuoka H, Alam KS, Yoshizato H, McEwen BS, Nakashima K (2000) A biphasic regulation of receptor mRNA expressions for growth hormone, glucocorticoid and mineralocorticoid in the rat dentate gyrus during acute stress. Brain Res 874:186-193.

Gouirand AM, Matuszewich L (2005) The effects of chronic unpredictable stress on male rats in the water maze. Physiol Behav 86:21-31.

Green S, Chambon P (1987) Oestradiol induction of a glucocorticoidresponsive gene by a chimaeric receptor. Nature 325:75-78.

Huang L, Killbride J, Rowan MJ, Anwyl R (1999) Activation of mGluRII induces LTD via activation of protein kinase $A$ and protein kinase $C$ in the dentate gyrus of the hippocampus in vitro. Neuropharmacology 38:73-83.

Kaufer D, Ogle WO, Pincus ZS, Clark KL, Nicholas AC, Dinkel KM, Dumas TC, Ferguson D, Lee AL, Winters MA, Sapolsky RM (2004) Restructuring the neuronal stress response with anti-glucocorticoid gene delivery. Nat Neurosci 7:947-953.

Kim JJ, Lee HJ, Han JS, Packard MG (2001) Amygdala is critical for stressinduced modulation of hippocampal long-term potentiation and learning. J Neurosci 21:5222-5228.

Kitraki E, Kremmyda O, Youlatos D, Alexis MN, Kittas C (2004) Genderdependent alterations in corticosteroid receptor status and spatial performance following 21 days of restraint stress. Neuroscience 125:47-55.

Landgraf R, Frank E, Aldag JM, Neumann ID, Sharer CA, Ren X, Terwilliger EF, Niwa M, Wigger A, Young LJ (2003) Viral vector-mediated gene transfer of the vole V1a vasopressin receptor in the rat septum: improved social discrimination and active social behaviour. Eur J Neurosci $18: 403-411$.

Luine V (2002) Sex differences in chronic stress effects on memory in rats. Stress 5:205-216.

Luine V, Rodriguez M (1994) Effects of estradiol on radial arm maze performance of young and aged rats. Behav Neural Biol 62:230-236.

McEwen BS (1999) Stress and hippocampal plasticity. Annu Rev Neurosci 22:105-122.

McEwen BS (2001a) Plasticity of the hippocampus: adaptation to chronic stress and allostatic load. Ann NY Acad Sci 933:265-277.

McEwen BS (2001b) Invited review: estrogens effects on the brain: multiple sites and molecular mechanisms. J Appl Physiol 91:2785-2801.

McEwen BS, Alves SE, Bulloch K, Weiland NG (1997) Ovarian steroids and the brain: implications for cognition and aging. Neurology 48:S8-S15.

McGaugh JL, Roozendaal B (2002) Role of adrenal stress hormones in forming lasting memories in the brain. Curr Opin Neurobiol 12:205-210.

McLaughlin J, Roozendaal B, Dumas T, Gupta A, Ajilore O, Hsieh J, Ho D, Lawrence M, McGaugh JL, Sapolsky R (2000) Sparing of neuronal function postseizure with gene therapy. Proc Natl Acad Sci USA 97:12804-12809.

Oitzl MS, de Kloet ER (1992) Selective corticosteroid antagonists modulate specific aspects of spatial orientation learning. Behav Neurosci 106:62-71.

Oitzl MS, van Haarst AD, de Kloet ER (1997) Behavioral and neuroendocrine responses controlled by the concerted action of central mineralocorticoid (MRS) and glucocorticoid receptors (GRS). Psychoneuroendocrinology 22:S87-S93.

Packard MG (1998) Posttraining estrogen and memory modulation. Horm Behav 34:126-139.

Packard MG, Kohlmaier JR, Alexander GM (1996) Posttraining intrahippocampal estradiol injections enhance spatial memory in male rats: interaction with cholinergic systems. Behav Neurosci 110:626-632.

Radecki DT, Brown LM, Martinez J, Teyler TJ (2005) BDNF protects against stress-induced impairments in spatial learning and memory and LTP. Hippocampus 15:246-253.

Roozendaal B (2003) Systems mediating acute glucocorticoid effects on memory consolidation and retrieval. Prog Neuropsychopharmacol Biol Psychiatry 27:1213-1223.

Roozendaal B, Quirarte GL, McGaugh JL (1997) Stress-activated hormonal 
systems and the regulation of memory storage. Ann NY Acad Sci 821:247-258.

Roozendaal B, Griffith QK, Buranday J, De Quervain DJ, McGaugh JL (2003) The hippocampus mediates glucocorticoid-induced impairment of spatial memory retrieval: dependence on the basolateral amygdala. Proc Natl Acad Sci USA 100:1328-1333.

Sandi C, Loscertales M, Guaza C (1997) Experience-dependent facilitating effect of corticosterone on spatial memory formation in the water maze. Eur J Neurosci 9:637-642.

Sandi C, Davies HA, Cordero MI, Rodriguez JJ, Popov VI, Stewart MG (2003) Rapid reversal of stress induced loss of synapses in CA3 of rat hippocampus following water maze training. Eur J Neurosci 17:2447-2456.

Sandstrom NJ, Williams CL (2001) Memory retention is modulated by acute estradiol and progesterone replacement. Behav Neurosci 115:384-393.

Sapolsky R, Brooke S, Stein-Behrens B (1995) Methodologic issues in studying glucocorticoid-induced damage to neurons. J Neurosci Methods 58:1-15.

Sapolsky RM, Krey LC, McEwen BS (1985) Prolonged glucocorticoid exposure reduces hippocampal neuron number: implications for aging. J Neurosci 5:1222-1227.

Sapolsky RM, Romero LM, Munck AU (2000) How do glucocorticoids influence stress responses? Integrating permissive, suppressive, stimulatory, and preparative actions. Endocr Rev 21:55-89.
Schaaf MJ, De Kloet ER, Vreugdenhil E (2000) Corticosterone effects on BDNF expression in the hippocampus. Implications for memory formation. Stress 3:201-208.

Sousa N, Almeida OF (2002) Corticosteroids: sculptors of the hippocampal formation. Rev Neurosci 13:59-84.

Sousa N, Lukoyanov NV, Madeira MD, Almeida OF, Paula-Barbosa MM (2000) Reorganization of the morphology of hippocampal neurites and synapses after stress-induced damage correlates with behavioral improvement. Neuroscience 97:253-266.

Stewart MG, Davies HA, Sandi C, Kraev IV, Rogachevsky VV, Peddie CJ, Rodriguez JJ, Cordero MI, Donohue HS, Gabbott PL, Popov VI (2005) Stress suppresses and learning induces plasticity in CA3 of rat hippocampus: a three-dimensional ultrastructural study of thorny excrescences and their postsynaptic densities. Neuroscience 131:43-54.

Vaher PR, Luine VN, Gould E, McEwen BS (1994) Effects of adrenalectomy on spatial memory performance and dentate gyrus morphology. Brain Res 656:71-78.

Woolley CS (1998) Estrogen-mediated structural and functional synaptic plasticity in the female rat hippocampus. Horm Behav 34:140-148.

Xavier GF, Oliveira-Filho FJ, Santos AM (1999) Dentate gyrus-selective colchicine lesion and disruption of performance in spatial tasks: difficulties in "place strategy" because of a lack of flexibility in the use of environmental cues? Hippocampus 9:668-681. 